\title{
CONCLUSION AND AGENDA FOR ITEM RESEARCH
}

\author{
ALEX C. W. FUNG* and ADRIE J. VISSCHER† \\ *Hong Kong Baptist University, Hong Kong \\ †University of Twente, The Netherlands
}

\begin{abstract}
This chapter integrates the previous chapters of this issue by presenting a holistic view on the development and implementation of SISs. This is done by discussing the elements of this innovation process. Subsequently, an agenda for future research in the field of ITEM is presented. Copyright $(C)$ 1996 Elsevier Science Ltd
\end{abstract}

\section{The SIS Innovation Process}

Reading through the chapters in this special issue, it becomes quite clear that school information systems are very complex. A consensus of the authors, it can be confidently concluded, is that developing and implementing a SIS is a multi-dimensional systemic socio-technical innovation process. This process is systemic in that it consists of a number of interrelated processes which are people-oriented as well as technical-oriented. Two aspects of integration are of concern in this innovation process. First, the technical design should aim at producing an integrated SIS for maximum organizational benefit; second, the change process has to be managed in an holistic manner such that the sub-processes are integrated.

Figure 8.1 is an illustration of this holistic concept where the SIS innovation process is viewed as a "working page" that contains five major "sub-pages" and one "linking page". The working page for SIS is coupled with the linking page of User-Developer Collaboration implying that user-developer collaboration is a prerequisite for success throughout the entire innovation process. The vertical and horizontal axes, representing respectively, the user dimension and the developer dimension, show this crucial interaction of the socio aspect in the process.

The other necessary factors in the innovation process - the technical - are contained in the sub-pages labelled, respectively, Philosophies \& Objectives, Strategies, Technical Design \& Development, Implementation, Monitoring \& Evaluation. These sub-pages are drawn as overlapping to indicate that they are interrelated in the whole process. Moreover, each sub-page has its own sub-components which are explained in the following paragraphs. Finally, the role of SIS researchers is also depicted in Figure 8.1. Depending on the background and interest of a 
researcher, different areas on the working page or linking page would be highlighted for study. The final section of this chapter refers to this aspect.

\section{SIS-Philosophies \& Objectives: Automating, Informating, Pedagogical}

Three levels of SIS objectives are discerned by the contributors to this special issue. These are related to the philosophy of applying IT in educational management. The first level relates to automating school administration, also referred to as datalogical. At this level the SIS mainly supports administrative functions.

The second level is informating or infological. At this level the SIS is managerial and provides support to decision making. The third level is pedagogical. Here the SIS can provide support to teaching and learning. Shifting from the first level to the other two is in fact a move from improved efficiency alone towards improving effectiveness. Unfortunately, current SISs

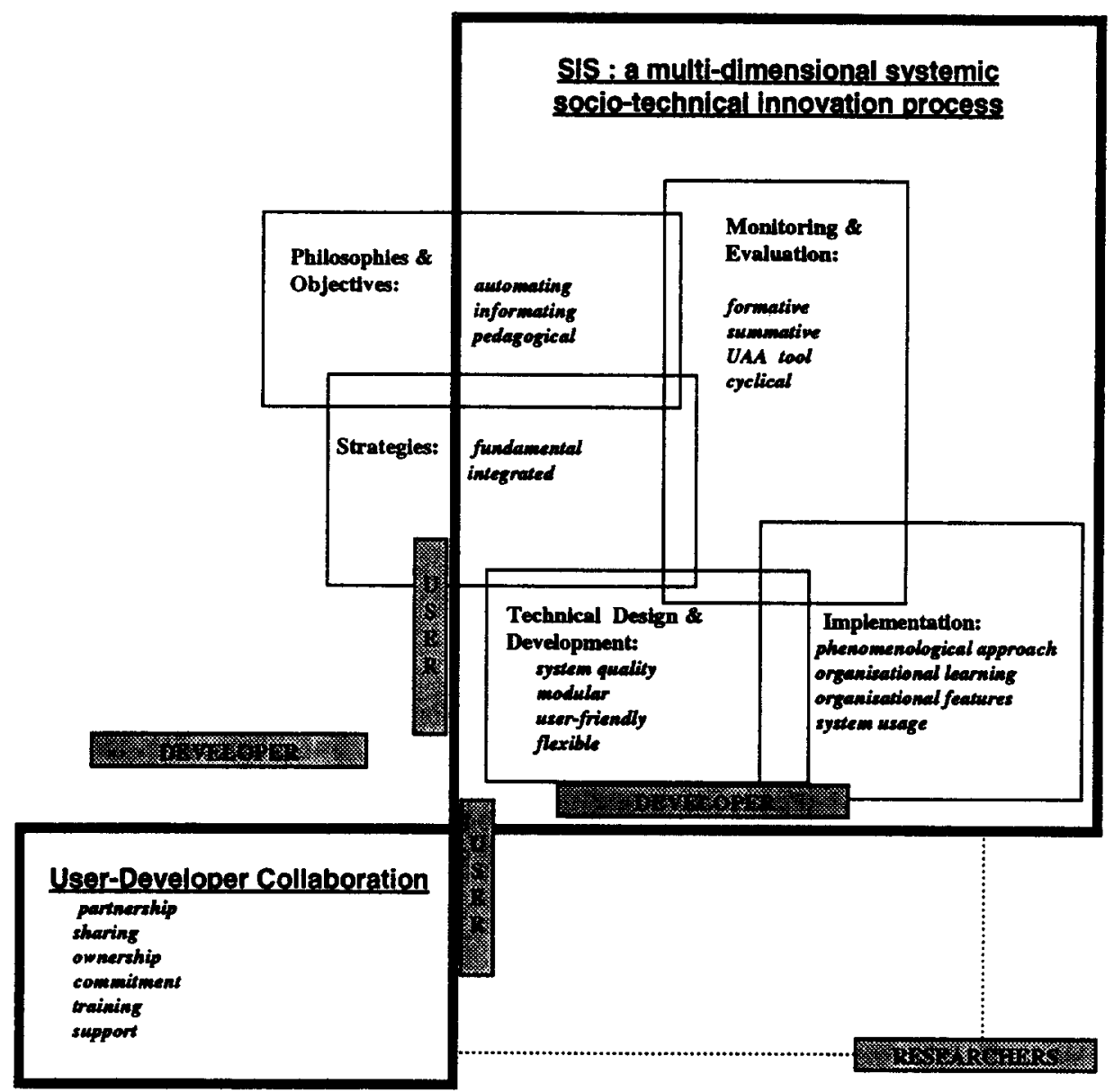

Figure 8.1. A holistic view of SIS-Innovation. 
are by and large only at the first level (see the chapters by Fung, Nolan \& Ayres, Visscher, and Wild). In the chapters by Fung and by Nolan, Ayres, Dunn, and McKinnon, the need to train better and develop more teachers and principals in the knowledge and skill for information technology in educational management is highlighted. This lack of IT expertise in schools is one reason why SISs, even with informating facilities as in MUSAC (Nolan \& Ayres' chapter), tend to be used only datalogically. A plausible inescapable path of SIS usage and development could be the sequential escalation of levels from the first to the third for the majority of educational institutions. One obvious determining factor for escalation could be the maturity and experience accumulated by individual schools using SISs.

Another factor, not so imminent, is whether schools have a culture supportive of school-based research for organizational development. It is argued here that schools without such a developmental culture would happily settle at the automating level. Schools with cultures that support school-based research for continual development will put greater value on a higher-level SIS in support of their search for improvement and increased pedagogical effectiveness. Only such schools would have the potential to develop and/or use SISs at the informating and pedagogical levels. This is an untested theory, however, that has yet to be substantiated by research.

\section{Strategies: Fundamental, Integrated}

The fundamental strategy (see Fung's chapter) advocates that the school organizational information needs should be the core of the SIS. A detailed study and analysis of the information requirements for managing the school efficiently and effectively should occur before designing and building an integrated system. Fung's chapter explains the concepts underlying this fundamental strategy and the integrated approach to SIS development. However, as Fung has remarked in his chapter, "a formal strategy is one thing; how that strategy is applied is another."

\section{Technical Design \& Development: System Quality, Modular, User-friendly, Flexible}

Nolan and Ayres' chapter provides a life example of the MUSAC system. It also validates how "good" SISs should be technically designed and developed by incorporating four attributes: integrated in architecture, modular in structure, user-friendly in interfacing, and flexible in anticipating changes. Furthermore, user definable data fields and report generators catering to individual needs will help preserve school identities without which user acceptance of the system will be minimal (see Wild's chapter). Such aspects are fundamental to system quality. SISs developed for regional or country use, in particular, must meet the challenge of standardization versus flexibility. A "good" system has to allow individual schools entry (i.e. adoption of the SIS) at different possible points which are compatible with the socio-technical environment of the schools. At the same time it must provide a developmental path for the schools for upgrading. The socio-technical environment of the schools is invariably context dependent and this component thus has a close relationship with the organizational features listed on the sub-page on Implementation. 
Implementation: Phenomenological Approach, Organizational Learning, Organizational Features, System Usage

In Chapter 5 a framework is developed for implementing computerized information systems. It is quite well accepted that implementing an innovation is a process and not simply a top-down event. As Nolan et al. state in their chapter, "To the extent that schools actually get the support and training they require, then the 'grass-roots,' bottom-up approach seems to offer the best means of developing, implementing and maintaining competent computer school information systems."

The concept of a phenomenological approach that emphasizes an understanding of the meaning for the participants in an innovation is an essential factor for implementation success. It is only when participants perceive the benefits of an innovation such as SIS and are ready to learn through the process of implementation that commitment and ownership can be generated. (Note the interrelationship here to the linking page on User-Developer Collaboration.) Implementation of a SIS is in effect the facilitation of the assimilation process of the SIS by the school. Furthermore, it is also an organizational learning process when the school goes through the phases of initiation, assimilation, and institutionalization during the innovation.

Visscher's chapter addresses the relationships between the way in which schools deal with information and the features of SISs. He shows that these by nature do not fit very well. This probably explains why SISs with the power to support higher order management functions (e.g. school review and strategic planning) are not used for those purposes. Visscher discusses ways in which higher levels of SIS usage can be achieved. This proves to be more than training school staff to "push the right button". First of all managers must be shown how valuable SISs can be for their policy-making activities. In most schools it moreover will require developing the whole school organization. Only then SIS usage for policy development purposes at all school organizational levels and in all areas of policy making can be achieved. Moreover, SIS information should be presented to school staff in such formats that match the way they operate.

\section{Monitoring \& Evaluation: Formative, Summative, UAA Tool, Cyclical}

Wild's chapter pinpoints the importance of user participation in developing and successfully implementing SISs. The lack of formative evaluation which could feed back valuable information during the innovation process is a major reason for SIS failures. The UAA (User Acceptance Audit) tool detailed in Wild's chapter should not be treated as a summative instrument only to be applied at the end of the implementation process. Formative evaluation should be applied throughout the entire SIS development and implementation process. Information and feedback so collected would serve as sign-posts for the SIS users and developers as they collaboratively go through the sub-pages illustrated in Figure 8.1. They would then be wellinformed without losing sight of "where they are now" and "where they are going."

\section{User-Developer Collaboration: Partnership, Sharing, Ownership, Commitment, Training, Support}

User participation in SIS development and implementation has been identified throughout the chapters of this special issue as a crucial element for success. However, user participation and 
involvement at the discretion of the developer would not be sufficient. In his chapter about the Hong Kong SAMs case, Fung has raised two questions: "Who is the genuine user?" and "Is the involvement genuine?"

It should be recognized that SIS developers and users have to truly collaborate as partners throughout the innovation process to get the maximum benefit. This generates ownership of the system by the users and also solicits their commitment to make the innovation a success. Partnership is the word indicating the interdependance of one on the other. Sharing ideas and expertise between the two parties, and feeding back constructive information are necessary at all the stages of SIS development and implementation, i.e. as they work through the sub-pages in Fig. 8.1.

Training and support, usually referred to as being provided to users, is undoubtedly necessary (see especially Fulmer's chapter). However, this is only one side of the coin. The IT experts have also to be conversant with school organizations, their information needs, and their cultures in order to develop good SISs. In this respect they also need training and support from school users. This spells out clearly again the need for partnership and collaboration which is the essence of the linking page.

\section{An Agenda for ITEM Research}

The use of information technology in educational management proves to have become a new and interesting area of research. Such research can provide insights into the degree to which ITEM has developed in various countries, the existing design and implementation problems, its impact, and ways in which problems can be overcome.

In Chapter 1 Visscher presents a framework for this special issue (see Blocks A to F in Figure 1.1). This framework is used here to formulate a proposal for future research in the field of ITEM. The framework is compared with the contents of the special issue to deduce what we do not know, but need to know to improve the status of ITEM. This analysis leads to the following research agenda.

\section{Block A: Design \& Development Strategy}

Fung has shown in Chapter 2 that designing SISs is a matter of making choices. Different design strategies result in SISs with different characteristics, that are appreciated by their users to different degrees, and, as a result, have different impacts. Fung clarifies the superiority of the fundamental design strategy. If this strategy is taken as a starting point, there are also various ways to operate within this strategy. For instance, how should the information requirements of school staff be determined, the SISs be designed and constructed, the process of change be managed, and to what degree and how should users participate in the design process? The advantages and disadvantages of alternative integrated strategies must be determined to identify which result in the most powerful SISs that schools appreciate and are willing to use. This may be done by carefully defining the characteristics of various integration strategies, designing and developing systems on the basis of each strategy, and subsequently evaluating the quality of the resulting SISs. 


\section{Block B: IS Quality}

Which characteristics do "good" SISs share and which types of support do they offer? Very little is known about the features of SISs that are appreciated and used intensely and the quality of support offered in terms of user friendliness and the usability of the produced information.

Nolan and Ayres refer in their chapter to some system quality indicators as mentioned by Fulmer. According to Fulmer, key attributes of good SISs are that they offer multiple input ports, alternative units of analysis for inquiry, variable types of data, choice of analytic processing, and variable access ports for the retrieval of data. Although these features are important they are quite global. They also are more a matter of opinion than based on empirical research. For instance, which types of data should SISs include and which options should users have to enter, retrieve, and analyze data? If we empirically can determine the features of successful SISs more precisely and find out why users value and benefit from certain systems more than others, we can improve existing systems and design better ones.

\section{Block C: Implementation Process Features}

A high quality SIS is no guarantee for successful implementation. Implementation process features prove to be crucial. It is known that certain factors like clarity of innovation goals, gradual innovation, user participation, innovation attitude, and training have an important influence on the progress of the implementation process. However, when asked for precise information on how to implement SISs one is forced to make no reply. A general conclusion must therefore be that we need more detailed studies of how the implementation process influences implementation outcomes.

Several authors in this special issue (Nolan et al., Fulmer, Visscher) have made a plea for training and development activities when introducing ITEM in schools. School staff have to learn new roles, procedures, knowledge, and skills. The meaning and value of SISs have to be explained, especially how SISs can be used for higher order management activities and school decision-making. School managers need to learn how these tools can be used in a technical way, but especially regarding the way to proceed once information has been retrieved. The extent to which principals are trained for this purpose is very limited and the knowledge concerning the best way to train them is even less. Most training is too narrow in scope because it focuses too much on keyboard skills and technical operation. Focused investigations into new methods of training educational managers in the use of SISs to enhance managerial capability which fully utilizes the SISs are urgently needed. A valuable way to do this may be to experiment with alternative training courses and evaluate the impact of these courses on manager performance over time. The contents, speed, and other instructional characteristics (e.g. problem-based, cooperative learning, experimental learning) should be varied to allow us to find out how more productive training methods can be designed.

In addition to identifying the needs and content for initial and in-service training, attention should be paid to how the skills and motivation of trainers influence their training success since the degree to which they are adequately prepared for the task at hand seems to be very decisive. Another important way to improve implementation quality concerns the use of the holistic evaluation techniques to assess various aspects of SIS implementation (see Wild in Chapter 7). Empirical research should clarify how the User Acceptance Audit (UAA) can be used to 
characterize the usability, utility and acceptability of the SIS to minimize or remove implementation blockages.

\section{Block D: Features of School Organizations}

More insight is also needed in the ways in which the nature of educational organizations interacts with SIS characteristics. Chapter 4 has shown some essential problems concerning the relationship between school staff behavior and the forms of support SISs can provide. It is generally assumed that SISs can produce much relevant information which previously was not available and the implication is that schools will benefit from these systems and this information.

Educational institutions have very specific organizational features, which have implications for how a SIS will be hosted by them. The fit of the interface between a SIS and the organizational context in which it is meant to be used is crucial. We should determine which organizational features play a role when implementing SISs and how we can reckon with them. For instance, how can the information which SISs produce be transformed in a way which stimulates its use and invites school staff to act?

Moreover, since full system usage requires organizational development, a great deal of effort has to be put in exploring ways to successfully accomplish organizational learning and development. How can teachers and principals be lifted to levels where the school staff fully benefits from SISs? How can schools be brought to stages of functioning at which school review and improvement are supported by the information which SISs can provide?

\section{Block E: Use of SIS}

Just as designers choose to produce a system with specific characteristics, school staff decide to make use of a SIS in a certain way. They do so

- with a certain intensity;

- to a certain extent for clerical purposes, and for school management activities;

- to a certain level for solving structured allocation problems, and for supporting decision making in unstructured contexts; and

- in a number of policy-making areas

Until now system usage has only been studied at a very superficial level, which has only vaguely provided us information on the features of system usage. We are therefore in need of objective, in depth, and diverse case studies of the actual use of SISs. In line with Nolan and Ayres (Chapter 3) system acquisition (the software schools possess) and patterns in existing information system use have to be investigated. The latter is the most important: how are programs used in the aforementioned ways? Nolan and Ayres' suggestion to explore exemplary practices in schools that do fully benefit from SISs is also worth mentioning since such studies can point to factors that are decisive for maximum benefit to be derived from the power of these systems.

\section{Block F: Impact}

Most research makes little attempt to assess the effectiveness of the management information system under investigation, let alone relate their findings of computer impact to overall 
effectiveness. Moreover, research on the organizational impact of system usage is seldom carried out; if any SIS impact is studied it is almost always done at individual or at some sub-unit level. Since SISs are meant to increase organizational effectiveness, an important evaluative question is whether the desired increase is actually realized. For example, to what extent is the work in school offices streamlined as a result of system use. How effective are the links between computer information, strategic planning, and organizational renewal and improvement?

In addition to the planned and desired effects, unplanned negative effects need to be studied. Examples include increased monotonous data entry and retrieval work, work fragmentation, and information overload.

Information on the impact of system use may best be gained by studying the effects of successful computer applications. This would prevent drawing false conclusions regarding the potential positive/negative impact of these systems. Moreover, when studying the impact of SISs researchers should not base their findings on user perceptions alone. Whenever possible they should make a pre-test/post-test comparison and relate it to the original design criteria for the SIS.

\section{Block A-F}

As mentioned earlier, the blocks in the Visscher model are supposed to be interrelated. The features of one block have consequences for one or more of the subsequent blocks. Empirical investigations are needed to establish the nature of these interrelationships. That research must clarify which design strategies produce high quality SISs; how information system usage is influenced by system quality, features of the implementation processes, and the schools themselves; and what effects are generated by SIS use.

\section{Research Methodology: A Closing Comment}

Many ITEM studies so far reported concern "one shot", small scale studies in which subjective user perceptions often are the basis for statements concerning system quality and the factors that are believed to influence system use and impact. We are in need of more longitudinal, large scale studies using objective measures to collect valid and reliable data on the qualities of alternative approaches for introducing and implementing SISs.

The increasing importance of ITEM in the field of education requires greater insight into the pros and cons of various ways to design, implement, and use SISs. Hopefully the directions for research proposed in this chapter will enable educational institutions in the near future to benefit more from the support these systems can provide. 